\title{
Diabetic Retinopathy: A Physician's View
}

\author{
J. D. WARD
}

Sheffield

Physicians and ophthalmologists are well aware of the devastating effect of diabetic retinopathy in producing so much visual loss in an age group within the working population. ${ }^{1}$ Until a more fundamental understanding of aetiological factors is available, minimisation of severe retinal damage will depend on adequate identification of those in need of laser treatment and attempts to control the blood glucose to as near normo glycaemic levels as possible. The former should be well within our ability to organise, the latter should be, but is fraught with many difficulties.

\section{Aetiology}

The factors which are considered to be important in the development of retinal damage leading to retinopathy are:

(1) Specific endotheleal cell disease with inhibition of capillary endotheleal cell growth or proliferation of these cells. ${ }^{2}$ Many growth factors have been identified which influence growth and development of these cells but the exact part they play in human disease is yet to be identified. ${ }^{3,4}$

(2) The influence of various hormones such as growth hormone $e^{5}$ and somatomedin ${ }^{6}$ are thought to have some part to play with reference to proliferation of vessels.

(3) Haemorrheological factors. ${ }^{7}$

(4) Abnormal blood flow and haemodynamic factors. ${ }^{8}$

(5) The polyol pathway. ${ }^{9}$

(6) Poor quality of blood glucose control.

Many of the features of the microvascular disease of diabetic retinopathy strongly suggest.problems with the endothelial cell although the evidence of the moment is indirect. Haemorrheological abnormalities have been reported in diabetic retinopathy over many years and clearly at the microvascular level, interaction of diseased endothelial cells and unusually sticky platelets or other abnormal coagulation factors could well be critical in producing serious vessel disease. However, as yet it is not known whether such coagulation abnormalities are primary, leading to vessel damage, or secondary to the widespread vascular damage so common in diabetes. By the time serious diabetic retinopathy is present, the patient has had diabetes for many years and other vascular disease is usually present. But presumably even at this stage a hypercoagulable state is likely to aggravate pathological change in the retinal vasculature. Long-term prospective studies are necessary to clarify the primary or secondary nature of these haemorrheological abnormalities.

Since it is agreed that many features of diabetic retinopathy are a response to ischaemia, there are indeed abnormalities of blood flow in the retina with areas of under perfusion and ischaemia. The venous distention strongly suggests arterio-venous shunting and this is in keeping with abnormalities of blood flow in the diabetic kidney ${ }^{10}$ and neuropathic foot, ${ }^{11}$ where in the latter, AV shunting is a wellestablished phenomenon.

\section{The Polyol Pathway}

For 10 years or more now a metabolic pathway has been intensively studied in relation to the development of the complications of diabetes-the polyol pathway. Most of the basic-animal work has been carried out in nerve, but there is evidence that metabolites are present in the kidney and retina. Directly related to hyperglycaemia, there is a marked 
accumulation in nerve of Sorbitol and Fructose. This is associated with depression of myo-inositol an essential precursor for phosphoinositide metabolism and this then leads to depression of the $\mathrm{NA}^{+} / \mathrm{K}^{+} A T P A S E$ enzyme activity. The integrity of this cycle is necessary for nerve energy requirements and it is now well-established that this altered metabolic state is directly responsible for the slowing of nerve conduction which is characteristic of diabetic nerve and for specific neuropathological changes in the nerve. This whole cycle is controlled by the enzyme aldose reductase responsible for reduction of glucose to sorbitol. Agents are now available-aldose reductase inhibitors (ARIs) which inhibit sorbitol accumulation and prevent the development of all of the metabolic, functional and structural abnormalities listed above-quite independently of the level of the blood glucose. Thus there is a potential to prevent or control a pathological metabolic pathway known to lead to tissue damage regardless of the blood glucose-an attractive proposition. Many studies in rats have confirmed the effectiveness of these agents but clinical efficacy in human diabetic neuropathy has yet to be established. In the many clincial trials so far reported only marginal clinical and functional improvements have so far been observed. It has to be said that at the stage of significant human clinical neuropathy nerve is severely diseased with loss of axons, demyelination and also microvascular disease leading to significant hypoxia. ${ }^{12}$ Moreover the metabolic pathway may no longer be relevant-in other words these drugs are being administered far too late in the natural history of the disease. To establish that they are of benefit long term preventative studies would be required which would be large, costly and long-lasting.

There is certainly good evidence that this polyol pathway is active in the retina, ${ }^{13}$ and studies have also been carried out with ARIs. Prevention of the pericyte loss observed in animal diabetic retinopathy has been reported with these agents. ${ }^{14}$ In galactosaemic dogs, where a similar myoinositol deficient pathway is produced as in diabetes, no such benefits have been observed following treatment with ARI. ${ }^{15}$ A number of studies are now under way in human subjects to assess the effect of ARIs on established early diabetic retinopathy over a four year period.

\section{Blood Glucose Control}

Clearly the generalised features of diabetic retinal damage as we observe it are unique to diabetes. Thus the blood sugar must have a major part to play. Over the decade much good circumstantial evidence has been produced strongly to suggest that there is a broad relationship between the quality of blood glucose control and the degree of retinopathy. However, many clinicians can quote well-controlled patients with retinopathy and poorlycontrolled subjects without, although this may partly reflect the relative inadequacy of our methods for assessing quality of blood glucose control. However, recently very convincing evidence, even approaching proof, has been produced from Wisconsin which puts heavy responsibilty on diabetologists to improve blood glucose control in all their patients. ${ }^{16}$ A total of 1,888 diabetic subjects underwent very detailed retinal examination by means of stereoscopic fundus photography and were followed for a four-year period, with glycosylated haemoglobin as the main measure of metabolic control. Allowing for age, sex, duration of diabetes and base-line retinopathy, the incidence of new retinopathy or the progression of established retinopathy was significantly related to elevation of the glycosylated haemoglobin. Proliferative retinopathy was common in younger subjects. This must be regarded as powerful evidence for the central role of hyperglycaemia in the development of retinal damage.

Paradoxically intensification of blood glucose control using insulin pumps, has in two studies shown deterioration in the state of the retina. ${ }^{22,23}$ After six to eight months of meticulous blood glucose control more cotton wool spots, intraretinal microvascular abnormalities and venous distension were seen compared to patients who had continued with their usual therapy. Fortunately after two years of therapy these signs of deterioration had reversed and there was little difference in retinal status between the two groups. It is thought that changes in blood flow following such meticulous control led to some areas of retina becoming more ischaemic. 
It is now well established from many clinical reports and from personal experience that in the presence of established retinopathy rapid improvement in blood glucose control may lead to devastating deterioration in the retinopathy. At least four cases are known personally to this author-eg: A 21 year old female with the most brittle form of diabetes had been an in patient for two years so severe was her glucose and acidaemic state. Blood glucose levels were constantly between $28-44$ $\mathrm{mmol} / \mathrm{l}$ despite massive doses of insulin and eventually, on account of extremely painful neuropathy, intravenous and intraperitoneal insulin was administered resulting in a fall of blood glucose to $10 \mathrm{mmol} / \mathrm{l}$. Within 10 days, the fundus having been normal to routine ophthalmascopic examination, the complaint of blurred vision led to the diagnosis of bilateral ischaemic maculopathy with some bleeding and new vessel formation. Fortunately laser treatment was successful.

There is now an important rule-do not attempt aggressively to improve blood glucose control in the presence of established retinopathy.

\section{Problems in Achieving Good Blood Glucose Control}

The achievement of good blood glucose control-i.e. as near normoglycaemia as possible, is more difficult in practice than might be supposed, particularly when large numbers of patients are to be cared for. There are many metabolic reasons for such difficulty. The subcutaneous root of administration is not the natural site for insulin absorption and indeed variable rates of absorption from day-to-day, in the same patient can make it impossible to write a logical prescription for insulin. As a result, many patients with the best of intentions, report wild swings in their own selfperformed blood glucose measures in the course of any one day. Normal regulatory hormonal responses to insulin and glucose levels are imparied in diabetes and the preparations of insulin available for administration, although highly purified, do not apparently suit a number of patients. Various methods of improving insulin delivery have been developed-e.g. insulin pumps, pens for easy transport of insulin, but at the end of the day, the insulin is still being injected under the skin. Future developments with devices to sense tissue glucose to help with self-adjustment of insulin dose or to control externally or implanted pumps may produce very significant improvements in our ability to control blood glucose. Pancreas transplantation and islet cell cultures with infusion of cells into the portal vein are areas which will perhaps develop in the future.

Of equal importance to metabolic problems are the personal problems that many people with diabetes face from day-to-day in the course of a life-time of this illness. The attitudes and personality of both patients and their professional advisers, their social situation and pressures of their lives with worry, concern, fear and lack of motivation all lead to great difficulty in carrying out the arduous task of constantly facing dietary restrictions, injections and blood sugar measurements. Fear is indeed an important factor in many people-fear of the long-term complications of diabetes and particularly fear of hypoglycaemia-it is now well-established that the more vigorous are the attempts to achieve normo-glycaemia, the higher will be the incidence of hypoglycaemia and hypoglycaemia is the most feared of the complications of diabetes. In trying to protect the patient from tissue damage by achieving good blood glucose control, the physician produces the very problem which they fear the most. Little wonder that many patients find themselves unable to produce the necessary effort and work to achieve good blood glucose control.

And finally, the manner in which we offer our diabetes services are so often inadequate and lead to total lack of motivation on the part of the patient. Visit crowded, dehumanised diabetic clinics where fit and younger people will be surrounded by the signs of the complications of diabetes, waiting a long time to be seen by different doctors and often criticised for inability to lose weight or achieve an adequate blood glucose level. Add to this lack. of time to explain the complexities of diabetes and its management and it is not surprising that many people feel overwhelmed. Much has to change to provide patients with support, education, time and clinical care that will allow many more to feel able to work 
harder at achieving this goal of near normo-glycaemi.

\section{Screening for Diabetic Retinopathy}

We now have an effective method of preventing the progression of retinal damage to such a state that visual loss occurs-namely laser photocoagulation. However, this can only be done effectively if the ophthalmologist is presented with patients at an early stage for more sophisticated assessment and application of appropriate therapy at the right time. In the United Kingdom the sole responsibility for identifying those patients requiring expert ophthalmological treatment rests with the diabetologist. Each diabetologist in a district should be responsible for the screening of the whole diabetic community. A district diabetes register is mandatory for both patients attending hospital based clinics and the estimated $50 \%$ who are under the care of their GP or indeed many of whom are receiving no care at all. The majority of this latter group are noninsulin dependent diabetic subjects, but they develop retinopathy as well. The establishment of a community register is a major administrative task.

Having identified the population to be screened we next ask what is the best method of screening. A number of options are available and studies of comparative costs and efficacy have been reported and many such studies are still under way. ${ }^{17,18}$

(a) Physician-diabetologist or general practitioner. With adequate training there is no reason why such physicians with the patient's eyes dilated cannot make an assessment of the retina as to whether there is disease present of a severity likely to need laser treatment or approaching such as time. Indeed it would be a bad day for medicine if consultant physicians in charge of a diabetes service could not make such an assessment with efficiency. GPs would require training and could well assist in this matter, either as clinical assistants or as one designated doctor per large group practice. In large well-staffed hospitals-often called Teaching Hospitals - this system of consultant and his senior junior staff screening is probably the method- of choice, however as the staff-patient ratio rises, other help may well be needed

(b) Cameras-mydriatic or non-mydriatic. There have been a number of studies into the usefulness of such cameras and they would seem to have a role in very busy centres or in country areas where such cameras can be transported to outlying districts and small towns. ${ }^{19,20}$ However, staff have to be trained, not only to take photographs but experts, technicians perhaps, will also have to review the films and then arrange for referral and treatment. These cameras are in no way perfect. Technically speaking the exact percentage of false positives and false negatives needs to be established. For physicians to be using such cameras seems quite unnecessary-they might just as well use an ophthalmoscope.

(c) There is absolutely no reason why opticians with motivation and training should not screen for diabetic retinopathy but they should be selected with care and of course will have to have an organisational back-up so that the results of their screening are notified to the diabetes services. ${ }^{21}$

All this is organisation not medicine. Organisation of a community register, the call and recall of patients to be examined by one or another of the methods described. Naturally there will have to be organisation to refer patients to appropriate ophthalmologists. Such organisation requires administration and clerical assistance which is usually totally lacking in many districts. In the future the aim of diabetes management in this country is that each health district should have a Diabetes Centre. Such a centre should be near, but not part of the Hospital Diabetic Clinic, should be available all day and every day and should co-ordinate hospital and general practitioner services and make best use of the diabetic specialist nurse who is now an integral part of diabetes management. Indeed it is likely that the most important development in the last decade has been the increasing involvement of the diabetes specialist nurse. With such a centre, patients can be offered open access for advice and treatment or planned sessions of a clinical and educational nature. The centre would be the obvious base from which to run the community register and regular screening would 
take place there, not only for retinopathy but for assessment of blood pressure, microproteinurea and the likelihood of foot problems.

This organisation around a Diabetes Centre is an essential part of the clinical care that should eventually lead to much improved patient acceptance of diabetes and the need to control the blood glucose and in the long term, a marked reduction in the morbid complications of diabetes.

\section{References}

${ }^{1}$ Foulds WS, MacCuish AC, Barrie T, et al. Diabetic retinopathy in the West of Scotland: Its detection and prevalence and the cost effectivness of a proposed screening programme. Health Bull (Edinburgh) 1983, 41: 318-26.

${ }^{2}$ Orlidge A and D'Amore PA: Inhibition of capillary endothelial cell growth by pericytes and smooth muscle cells. J Cell Biol 1987; 105: 1445-62.

${ }^{3}$ Baird A and Ling N: Fibroblast growth factors are present in extracellular matrix produced by endothelial cell in vitro. Implications for a role of Heparinase-like enzymes in the neovascular response. Biochem Biophys Res Communication 1987, 142: 428-35.

${ }^{4}$ Hyer SL, Sharp PS, Brooks RA, Burrin JM, Koner EM: Serum IGF-1. Concentration in diabetic retinopathy. Diab Med 1988, 5: 356-60.

${ }^{5}$ Lundbaek K, Christensen NJ, Jensen VA, et al. Diabetes, diabetic angiography and growth hormone. Lancet 1970, 2: 131-3.

${ }^{6}$ Ashton IK, Dornan TL, Pocock AF, et al. Plasma somatomedin activity and diabetic retinopathy. Clin Endocrinol 1983, 19: 105-9.

${ }^{7}$ Colwell JA: Platelets in diabetic retinopathy. In: Little HL, Jack RL, Patz A, Forsham PH. Eds. Diabetic retinopathy. New York, Thieme Stratton, 1983. p.119.

${ }^{8}$ Fallon TJ, Chowiencyzk P, Koner EM: Measurement of retinal blood flow in diabetes by the blue light entopic phenomenon. $\mathrm{Br} J$ Ophthalmol 1986, 70: 43-6.

${ }^{9}$ Greene DA, Lattimer SA, Sima AAF: Sorbitol phosphoinositides and sodium potassium ATPASE in the pathogenesis of diabetic complications. N Engl J Med 1987, 316: 599-606.

${ }^{10}$ Christensen JS, Gammelgaard J, Frandsen M, Par- ving $\mathrm{HH}$ : Increased kidney size, glomeral infiltration rate and renal plasma flow in short term insulin dependent diabetics. Diabetologia 1981, 20: $451-6$.

${ }^{11}$ Ward JD, Boulton AJM, Simms JM, Sandler DA, Knight GE 1983: Venous distension in the diabetic neuropathic foot (Physical sign of arteriovenous shunting). J Roy Soc Med 1983, 76: 1011-14.

${ }^{12}$ Newrick PG, Wilson AJ, Jakubowski J, Ward JD: Sural nerve oxygen intention in diabetes. $\mathrm{Br} \mathrm{Med}$ J 1986, 293: 1053-4.

${ }^{13}$ Akagi Y, Yajima Y, Kabor PF, Kuwabara T, Kinoshita JH: Localisation of aldose reductase in the human eye. Diabetes 1984, 33: 562-6.

${ }^{14}$ Kabor PF, Kinshita JH, Sharpless NE 1985: Aldose reductase inhibition: A potential new class of agents for pharmacological control of certain diabetic complications. J Med Chem 1985, 28: 841-9.

${ }^{15}$ Engerman RL, Kern TS: Effect of sorbinil on retinopathy in long term galactosaemic dogs. Diabetes 1989, 38(2): 24a.

${ }^{16}$ Klein R, Klein BEK, Scot EM, Davis MD, DeMets DL: Glycosylated haemoglobin predicts the incidence and progression of diabetic retinopathy. $J$ Am Med Ass 1988, 260: 2864-71.

${ }^{17}$ Waugh NR, Ellingford A, Scott SD: Screening for diabetic retinopathy. Options and cost effectiveness. Practical Diabetes 1986, 3: 30-1.

${ }^{18}$ Hill RD: Primary health care screening programme for diabetic eye disease. Diabetologia 1981, 20: 670-8.

${ }^{19}$ Burns-Cox CJ, Dean-Hart JC: Advantages and costs of screening with non-mydriatic fundus camera. Practical Diabetes 1986, 3: 166.

${ }^{20}$ Ryder REJ, Young S, Vora J, Atiea J, Owens DR, Hayes PM: Screening for diabetic retinopathy using polaroid retinal photography through undilated pupils. Practical Diabetes 1985, 2: 34-40.

${ }^{21}$ Burns-Cox CJ, Dean Hart CJ: Screening of diabetic retinopathy by opthalmic opticians. $B$ Med $J$ 1985, 290: 1052-4.

${ }^{22}$ Lauritzen T, Frost-Larsen K, Larsen HW, Deckert T: The steno study group. Effect of one year normal blood glucose levels on retinopathy on insulin dependent diabetics. Lancet 1983, i: 200-4.

${ }^{23}$ The Kroc Collaborative Study Group: Blood glucose control and the evolution of diabetic retinopathy and albuminuria. N Engl J Med 1984, 311: $365-72$. 